\title{
Kulturstiftung des Bundes fördert Klima-Pilotprojekt in Kultureinrichtungen
}

https://doi.org/10.1515/bd-2021-0042

Die Kulturstiftung des Bundes hat mit „Klimabilanzen in Kulturinstitutionen“ ein Pilotprojekt initiiert, das 19 Kultureinrichtungen bundesweit dabei unterstützt hat, eine Klimabilanz zu erstellen und den eigenen $\mathrm{CO}_{2}$-Fußabdruck zu ermitteln. Ziel war es, modellhaft den Prozess der Klimabilanzerstellung im Kulturbereich $\mathrm{zu}$ erproben, um Kultureinrichtungen konkrete Instrumente zur Ermittlung der Emissionswerte an die Hand zu geben und Möglichkeiten auf dem Weg zur Klimaneutralität aufzuzeigen.

In einem viermonatigen Prozess erhielten die teilnehmenden Einrichtungen sowohl Unterstützung bei der Bilanzierung als auch ein spezifisches Klima-Coaching, um den Wissenstransfer im Haus über ein verbessertes Umwelthandeln zu gestalten. Jedes Haus konnte auf diese Weise Zahlen und Werte ermitteln, die nun gleichzeitig Standortbestimmung und Grundlage für die individuelle Strategieentwicklung sind. Nicht der Konkurrenzgedanke, sondern das gemeinsame Lernen und das Analysieren der individuellen Ausgangssituationen standen im Projekt im Fokus. Für die Kulturstiftung des Bundes geht es in diesem Vorhaben zudem um die Frage, wie ökologische Nachhaltigkeit in einem größeren Maßstab in ihrem Fördersystem verankert werden kann. Nicht zuletzt hat sie im Rahmen des Projekts auch selbst eine Klimabilanz für ihre Standorte in Halle und Berlin erstellt. Die Stiftung stellte für das Projekt insgesamt 120.000 Euro zur Verfügung.

Hortensia Völckers, Künstlerische Direktorin der Kulturstiftung des Bundes: „Wir müssen die Bedingungen künstlerischer Arbeit in Zeiten des Klimawandels gemeinsam diskutieren, überprüfen und neu gestalten. Jede Veränderung, die auf Dauer wirken soll, braucht eine Standortbestimmung. Die positive Resonanz der 19 beteiligten Einrichtungen bestärkt uns, den eingeschlagenen Weg programmatisch weiter zu verfolgen.“

Das Pilotprojekt wurde im Verbund umgesetzt. Ein solches „Konvoi-Verfahren" ermöglicht, dass die beteiligten Organisationen, begleitet durch Beraterinnen und Berater, ihre Erfahrungen teilen und voneinander lernen können. Die Pilotgruppe wurde durch die Kulturstiftung des Bundes mit dem Ziel zusammengestellt, eine möglichst große Bandbreite an Kultureinrichtungen aus dem Bundesgebiet abzubilden. Berücksichtigt wurden verschiedene Sparten, Einrichtungsgrößen, Standortbedingungen und Vorkenntnisse der Institutionen. 
Mit dem von der Europäischen Kommission vorgelegten Green New Deal sollen die Netto-Treibhausgasemissionen in der EU bis 2050 auf Null gesenkt werden. Deutschlands Ziel ist es, die Emissionen bis 2030 gegenüber 1990 um 55 Prozent zu senken. Das sind große Ziele, die nur erreicht werden können, wenn sie als gesamtgesellschaftliche Aufgabe verstanden werden und dieser Transformationsprozess auch im Kulturbereich stattfindet.

Konkret ging es in den Einrichtungen um folgende Fragen: Wie groß ist der $\mathrm{CO}_{2}$-Fußabdruck eines Hauses, auf welche Aktivitätsfelder verteilen sich die Emissionen und wo können wesentliche Verbesserungen erzielt werden? Im Pilotprojekt wurden die Klimabilanzen nach dem internationalen Berichtsstandard des „Greenhouse Gas Protocol (GHG)“ erarbeitet, das zwischen direkten, indirekten und vor- bzw. nachgelagerten Emissionen unterscheidet. Demgemäß wurden insbesondere die folgenden Emissionsquellen für das Jahr 2019 untersucht: Infrastruktur (Strom- und Wasserverbrauch, Wärme/Kälte, Abfall), Mobilität (der Mitarbeitenden, der Besucher, der Künstler und der Gegenstände/Transporte) sowie Beschaffung (für Werkstätten, Verwaltung oder Gastronomie). Mittels dieser Daten erhält jedes Haus eine Aufstellung und Analyse seiner Zahlen, um relevante Einsparpotenziale identifizieren und Handlungsempfehlungen daraus ableiten zu können.

Bis Ende April 2021 werden die Erkenntnisse aus dem Pilotprojekt ausgewertet und für eine Online-Publikation aufbereitet. Neben hilfreichen Tipps u. a. zu ersten Schritten bei der Klimabilanzierung, werden hier auch Fachartikel und Grafiken veröffentlicht. Die Publikation steht ab Mitte Mai zum kostenfreien Download auf der Website der Kulturstiftung des Bundes bereit.

Folgenden Einrichtungen haben sich am Projekt beteiligt:

- Deutsches Nationaltheater Weimar

- Gedenkstätte Sachsenhausen

- Kampnagel Internationale Kulturfabrik, Hamburg

- Konzerthaus Berlin

- Kunsthalle Rostock

- Künstlerhaus Mousonturm, Frankfurt

- Kunstverein Hannover

- Lenbachhaus, München

- Museum Folkwang, Essen

- Saarländisches Staatstheater, Saarbrücken

- Schaubühne am Lehniner Platz, Berlin

- Stadtbibliothek Berlin-Pankow

- Stadtbücherei Norderstedt

- Staatliche Kunstsammlungen Dresden

- Staatsphilharmonie Rheinland-Pfalz, Ludwigshafen 
- Staatsschauspiel Dresden

- Staatstheater Darmstadt

- tanzhaus nrw, Düsseldorf

- Zentrum für Kunst und Medien, Karlsruhe

\section{Weitere Informationen im Internet:}

- Projekt: http://bit.ly/klimabilanzen

- Datenschutz: www.kulturstiftung-des-bundes.de/datenschutz

\section{Kontakt:}

Friederike Tappe-Hornbostel

Leiterin Kommunikation

Kulturstiftung des Bundes

Franckeplatz 2 | 06110 Halle/Saale

Tel.: + 49 (0)345/2997-120 | Fax: + 49 (0)345/2997-300

E-Mail: presse@kulturstiftung-bund.de | URL: www.kulturstiftung-bund.de

Die Kulturstiftung des Bundes wird gefördert von

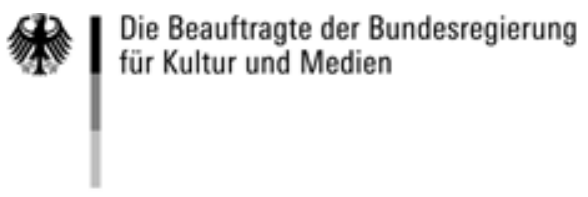

aufgrund eines Beschlusses des Deutschen Bundestages. 